\title{
Thermostructural characterization and structural elastic property optimization of novel high luminosity LHC collimation materials at CERN
}

\author{
M. Borg, ${ }^{1}$ A. Bertarelli, ${ }^{2}$ F. Carra, ${ }^{2}$ P. Gradassi, ${ }^{2}$ J. Guardia-Valenzuela, ${ }^{2}$ M. Guinchard, ${ }^{2}$ \\ G. Arnau Izquierdo, ${ }^{2}$ P. Mollicone, ${ }^{1, *}$ O. Sacristan-de-Frutos, ${ }^{2}$ and N. Sammut ${ }^{1}$ \\ ${ }^{1}$ University of Malta, Msida MSD 2080, Malta \\ ${ }^{2}$ CERN, CH-1211 Geneve 23, Switzerland
}

(Received 20 June 2017; published 5 March 2018)

\begin{abstract}
The CERN Large Hadron Collider is currently being upgraded to operate at a stored beam energy of 680 MJ through the High Luminosity upgrade. The LHC performance is dependent on the functionality of beam collimation systems, essential for safe beam cleaning and machine protection. A dedicated beam experiment at the CERN High Radiation to Materials facility is created under the HRMT-23 experimental campaign. This experiment investigates the behavior of three collimation jaws having novel composite absorbers made of copper diamond, molybdenum carbide graphite, and carbon fiber carbon, experiencing accidental scenarios involving the direct beam impact on the material. Material characterization is imperative for the design, execution, and analysis of such experiments. This paper presents new data and analysis of the thermostructural characteristics of some of the absorber materials commissioned within CERN facilities. In turn, characterized elastic properties are optimized through the development and implementation of a mixed numerical-experimental optimization technique.
\end{abstract}

DOI: 10.1103/PhysRevAccelBeams.21.031001

\section{INTRODUCTION}

The LHC is currently being upgraded to operate at twice the stored beam energy of previous runs through the high luminosity LHC (HL-LHC) upgrade [1,2]. Operating at unprecedented stored beam energies necessitates improved machine protection and response assessment of beam intercepting devices (BIDs). Collimators, as shown in Fig. 1, form a major part of the BIDs and protective system. These ensure that the LHC running conditions are stable with minimal damaging events, as has been successfully implemented during the LHC first run. As beam bunches circulate within the LHC ring, a slow diffusion of particles out of the beam core is instigated via long range beam-beam effects, intrabeam scattering, and orbit feedback systems, amongst others [3-7]. Particles at the edge of the spatial distribution tend to escape from the intended beam trajectory, consequently forming a beam halo.

The LHC collimation system targets over $99 \%$ efficient regular and irregular beam halo cleaning during the entire

\footnotetext{
*Corresponding author. pierluigi.mollicone@um.edu.mt
}

Published by the American Physical Society under the terms of the Creative Commons Attribution 4.0 International license. Further distribution of this work must maintain attribution to the author(s) and the published article's title, journal citation, and DOI. beam cycle at all stages of operation [9]. The hierarchical collimation structure consists of a multistage beam cleaning process, ranked according to beam proximity. The setup consists of primary collimator jaws positioned closest to the beam, followed by secondary collimators (TCS) retracted farther away from the beam and finally, tertiary collimators found at the farthest position from the beam. Although carbon fiber composite (CFC) presents an astonishing robustness in case of particle beam impact, its poor electrical conductivity impedes its use in primary and secondary

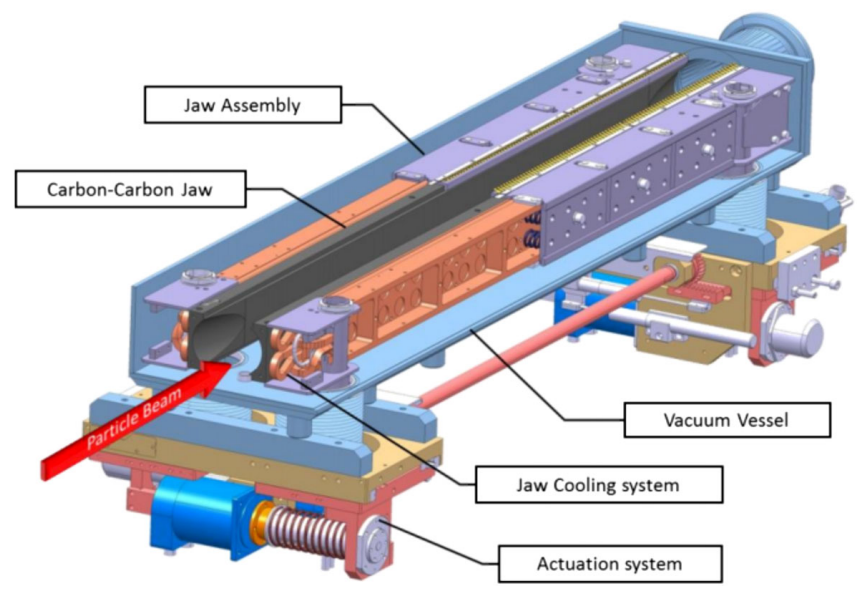

FIG. 1. Cutaway of a secondary collimator as a beam intercepting device with the active component made of a fiber-reinforced carbon jaw [8]. 
collimators due to the high rf impedance generated on the particle beams. On the other hand, the high-density tungsten alloy adopted in tertiary collimators cannot sustain the accidental impact scenarios of HL-LHC [10]. The molybdenum-graphite composite recently developed at CERN and Brevetti Bizz (Italy) [11] is proposed as a replacement of CFC for primary and secondary collimators, balancing a good thermomechanical robustness and stability during normal operation with a good electrical conductivity. On the other hand, copper diamond is proposed as a much higher resistant replacement for the tungsten alloy adopted in tertiary collimators.

The collimator materials, from the thermomechanical point of view, should possess high thermal conductivity, specific heat, melting temperature, and ductility; at the same time, coefficient of thermal expansion and density should be minimized. Additional requirements include high electrical conductivity, machinability, radiation hardness, and low magnetic susceptibility [12]. To classify and rank potential materials against this large number of requirements, it is useful to introduce figures of merit which permit several material properties related to a specific requirement to be condensed into a single indicator: the higher the figure of merit, the better the material performance against that specific requirement [13].

An index called the thermomechanical robustness index (TRI), is proposed to assess the material robustness against particle beam impacts. Given that thermal shock problems are, to a large extent, governed by the thermal deformation induced by a sudden temperature increase, it appears reasonable to base this index on the ratio between material admissible strain or strain to failure $\varepsilon_{\text {adm }}$ and the actual working strain as defined by Eq. (1):

$$
\mathrm{TRI}=\frac{\varepsilon_{\mathrm{adm}}}{\varepsilon_{\mathrm{ref}}} \cdot\left(\frac{T_{m}}{\Delta T_{q}}-1\right)^{m},
$$

where $\varepsilon_{\text {ref }}$ and $\Delta T_{q}$ are the strain and the temperature increase generated by a reference energy deposition, $T_{m}$ is the melting (or degradation) temperature, and $m$ is a coefficient related to the material loss of strength with temperature increase.

Another index, called the thermal stability index (TSI) provides an indication of the ability of the material to maintain the geometrical stability of the component under steady-state particle losses. This is particularly important for components such as collimators and long absorbers, which are required to interact with the halo of the particle, maintaining their longitudinal straightness to a fraction of a beam transverse sigma. TSI is defined by Eq. (2) as

$$
\mathrm{TSI}=\frac{\lambda X_{g}}{(\mathrm{CTE}) C_{S} \rho}
$$

where CTE is the coefficient of thermal expansion [defined later in Eq. (9)], $\rho$ the density, $\lambda$ the thermal conductivity, $X_{g}$ the geometric radiation length, and $C_{s}$ a material constant related to thermal stability.

Finally, the rf impedance index RFI is defined by Eq. (3) as

$$
\mathrm{RFI}=\sqrt{\frac{\sigma}{\mu}},
$$

where $\sigma$ is the electrical conductivity and $\mu$ the magnetic permeability.

Table I provides these figures of merit for $\mathrm{CFC}$ and tungsten alloy currently adopted in LHC collimators, and the requirements for HL-LHC.

The latest efforts to optimize the operation of collimators target novel absorbent insert composites that can sustain severe beam impacts. The high-energy particle-matter interactions impart a nonuniform temperature increase in the impacted structure, subsequently leading to physical deformations and stress to the possible detriment of the structure functionality. To analyze this behavior, tests are carried out within the High Radiation to Materials (HiRadMat) testing facility at CERN, which allows submitting targets to intense proton or ion beam impacts [14]. Distinct experiments are attempting to replicate collimation jaw behavior exposed to direct interaction with high-energy particles, while taking into account the jaw robustness, performance, fabrication, installation, beam operation, and machine maintenance. In conjunction with the design, running, and analysis of any experiment such as HiRadMat, knowledge of the thermophysical temperature-dependent properties of absorbers, experiencing energy deposition like in the HL-LHC, is essential to allow representative material behavior modeling. In light of this, several

TABLE I. Figures of merit for CFC and tungsten alloy currently adopted in LHC collimators, and the requirements for HL-LHC.

\begin{tabular}{lcccc}
\hline \hline & $\begin{array}{c}\text { Primary/secondary HL-LHC } \\
\text { collimator minimum } \\
\text { target }\end{array}$ & $\begin{array}{c}\text { Tertiary HL-LHC } \\
\text { collimator minimum } \\
\text { target }\end{array}$ & Carbon-carbon & $\begin{array}{c}\text { Tungsten heavy } \\
\text { alloy }\end{array}$ \\
\hline TRI & 200 & 5 & $800-1200$ & 0.5 \\
TSI & 40 & 0.1 & 45 & 0.14 \\
RFI & 1 & 2.5 & 0.38 & 2.9 \\
\hline \hline
\end{tabular}




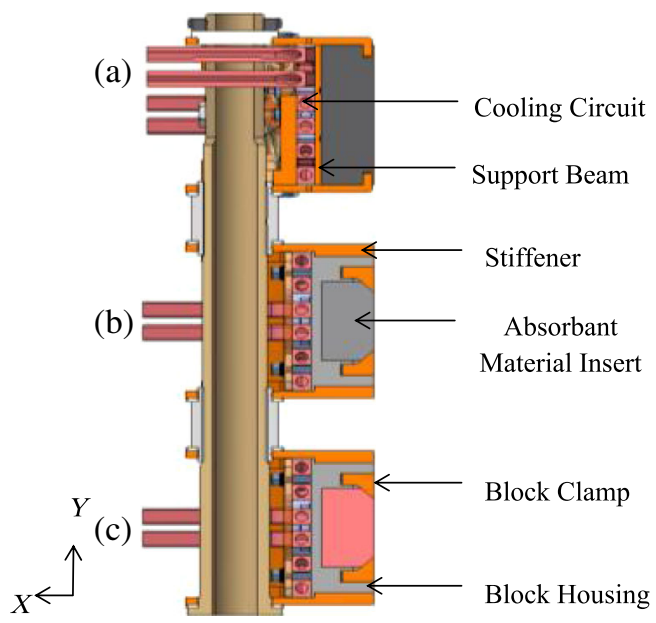

FIG. 2. Internal HRMT-23 jaw $X-Y$ cross-sectional view for (a) TCSP, (b) TCSPM-M, and (c) TCSPM-C [16].

experiments have been commissioned at the HiRadMat facility under the HRMT-23 experimental campaign.

This paper investigates the properties of the materials of the three jaws analyzed in the HRMT-23 campaign. The aim of this campaign is to investigate the thermomechanical properties of three independent jaws in order to guarantee robustness and geometric stability against beam impact, thus qualifying or disqualifying them for use in future fullscale HL-LHC prototypes. The jaws in question consist of two TCSPM and one TCSP jaw, generally distinguished by variations in the absorbent insert considered (Fig. 2). The main features of each jaw consist of the absorbers located at the jaw center, experiencing direct interaction with the particle beam. The inserts consists of novel copper diamond ( $\mathrm{CuCD})$, molybdenum carbide graphite (MoGr), and carbon fiber carbon composites, behaving in an isotropic, transversely isotropic, and orthotropic manner, respectively, as determined by Mariani [15].

$\mathrm{CuCD}$ consists of a diamond reinforced metal matrix composite developed to optimize the thermal management properties of both the copper and diamond constituent. $\mathrm{CuCD}$ is volumetrically composed of $60 \%$ diamond powder at a diameter of $\sim 100 \mu \mathrm{m}$ for $90 \%$ of the powder and a rougher diameter of 325 to $400 \mu \mathrm{m}$ for the remaining $10 \%$ [11]. The composite is developed by means of hot processing powder technologies utilizing several stages of hot pressing and subsequent heat treatments, to provide an inherently isotropic and stress relieved composite. The composite depicts a rough structure where the weakest element is the interface between the copper matrix and diamond particles, as shown by fracture surfaces, observed as prominent voids. These voids indicate preferential fracture across the brittle diamond interfaces, without visible signs of plastic deformation of the copper matrix. $\mathrm{CuCD}$ is particularly appealing for use in LHC collimators due to its ability to maximize geometric stability and transient shock response, while exhibiting high electrical and thermal conductivities as well as low coefficient of thermal expansion and low densities. The lack of diamond degradation and relatively low densities also allows for a smoother energy deposition profile, while generating high beam impact strength, essential for superior resistance to beam impacts. However, $\mathrm{CuCD}$ is limited by the low melting temperature of the copper matrix, especially when compared to other absorbent inserts, reducing overall robustness.

MoGr is a graphitic matrix composite, consisting mostly of spheroidal graphitic flakes. It exhibits the highest chemical purity and degree of graphitization of lamellar graphite flakes with a diameter of $45 \mu \mathrm{m}$, having a distinctly regular and round shape that assists in powder compaction and reduces the final anisotropy of the composite. The remainder of the composite is volumetrically composed of 4.5 vol \% molybdenum. Spark plasma sintering is carried out with processing temperatures ranging from 1700 to $2600{ }^{\circ} \mathrm{C}$. Because of the raw purely metallic molybdenum and graphitic powders being chemically affine to one another, sintering creates a highly integrated composite. During sintering recrystallization of graphitic grains also occurs and, because of the pressing process, graphite orients itself parallel to the sintering plate. This in turn creates an inherently transversely isotropic composite with preferential properties along the directions parallel to the sintering plane [11].

Tests carried out within the HRMT facility in previous years utilized $\mathrm{CFC}$ as a control material having previously survived under full LHC beam impacts. CFC is a commercial carbon-matrix composite consisting of two dimensional planes of short carbon fiber reinforcements embedded within a graphitic matrix. Short carbon fibers are randomly dispersed, creating a homogeneous layer along the plane of deposition. Several carbon fiber planes are stacked on top of one another and bundled into homogeneous layers in the perpendicular direction to the fiber orientation, creating a composite with outstanding mechanical and thermal properties with respect to isotropic graphite. After successive layering and hot pressing cycles, several stages of heat treatment follow. Subsequently, bar extrusion is carried out to increase the final density and provide a higher flexural strength between the two plane directions, resulting in an inherently orthotropic composite. CFC has been chosen for application in the LHC collimation system due to its good resistance to high thermal shocks and low CTE. This ensures high geometric stability and robustness, as required by the design specifications. CFC maintains high strength and low coefficient of thermal expansion at temperatures above $2000^{\circ} \mathrm{C}$ and displays excellent thermal shock resistance.

The orthogonal coordinate system shown in Fig. 2 for the HRMT-23 jaws is maintained for the remainder of this work, where $X, Y$ and $Z$ represent transversal $(T)$, 
perpendicular $(P)$, and longitudinal $(L)$ directions respectively. TCSPM jaws are fairly similar and mainly distinguished by the differing inserts considered, where $\mathrm{CuCD}$ and MoGr are utilized for TCSPM-C and TCSPM-M jaws, respectively. The final TCSP jaw exhibits distinct structural features in comparison to TCSPM jaws, while containing $\mathrm{CFC}$ inserts.

\section{THERMAL CHARACTERIZATION}

The thermal characterization campaign considers three techniques: laser flash analysis (LFA), differential scanning calorimetry (DSC), and dilatometry (DIL) with specimens having the dimensions denoted in Fig. 3. The experimental setup has been calibrated as per the required standards, utilizing thermal gauges with an accuracy of $\pm 0.05 \%$.

\section{A. Laser flash analysis}

The transient LFA method introduced by Parker et al. [17], based on the theory of Carslaw and Jaeger [18], is aimed at measuring diffusivity of a material specimen under noncontacting analysis. The experimental setup consists of a NETZSCH LFA 427 system, operating within a thermal range of 22 to $2000^{\circ} \mathrm{C}$ according to the ASTM E1461-13 standard [19]. The plane parallel disc specimens used in the test are spray coated with graphitic deposition, which improves the emissivity behavior prior to insertion within an inert argon atmosphere at pressures up to $10^{-5}$ mbar with a gas flow rate of $50 \mathrm{ml} / \mathrm{min}$. The pure gas operating environment is considered, to prevent unwanted corrosive reactions between specimen and setup components, while operating under isothermal $(\delta T / \delta t<0.1 \mathrm{~K} / \mathrm{min})$ conditions.

(a)

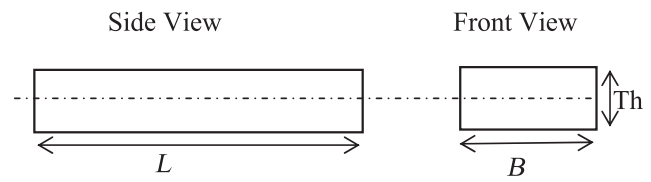

(b)

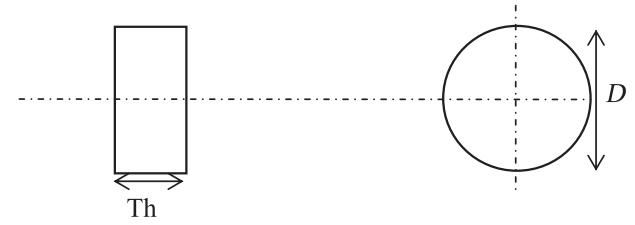

(c)
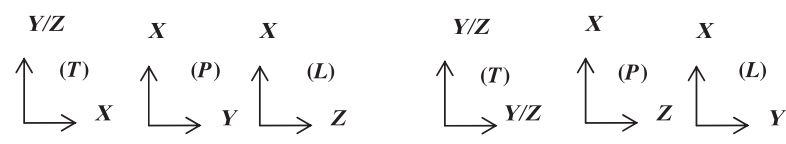

FIG. 3. Schematic experiment of (a) rectangular, (b) cylindrical specimen geometries, and (c) corresponding orthogonal coordinate system with $X, Y$, and $Z$ representing transversal $(T)$, perpendicular $(P)$, and longitudinal $(L)$ orientations, respectively.
A nitrogen-filled protective cooling tube is used while the surrounding furnace is activated. After the specimen reaches a predetermined temperature, a high-intensity light laser energy pulse is fired towards the bottom side of the sample, inducing a temperature rise. The thermal rise induced as a function of time is recorded by a noncontact infrared detector on the opposing sample side, recording the time-dependent energy transmission rate.

Thermal diffusivity $(\alpha)$ is obtained from the empirical relation developed by Parker et al. [17] using the mathematical solution of Carslaw and Jaeger [18]. For Eq. (4), $t_{1 / 2}$ represents the time in which the temperature rise on the specimen side opposite to where heat is applied reaches half of the maximum temperature, and $c$ represents specimen thickness. Thermal conductivity $(k)$ is determined via the empirical relation in Eq. (5), as a function of temperature [20],

$$
\begin{aligned}
& \alpha=-\frac{\ln (0.25)}{\pi^{2}} \frac{c^{2}}{t_{1 / 2}}, \\
& k=\alpha \rho C_{p} .
\end{aligned}
$$

\section{B. Differential scanning calorimetry}

Differential scanning calotimetry (DSC) is an experimental technique used to determine the specific heat capacity of a material. The technique consists of measuring the energy required to obtain a near-to-zero temperature difference between the specimen being tested and a reference inert specimen, which has known properties. The reference specimen has properties that are well known for the tested temperature range. Since the two samples experience the same thermal history, the specific heat for the unknown specimen under investigation can be calculated. Testing is set up in accordance with the ASTM E1269-11 standard [21] functioning within a thermal range of $40{ }^{\circ} \mathrm{C}$ to $500{ }^{\circ} \mathrm{C}$. Above $500^{\circ} \mathrm{C}$, the specific heat is assumed to remain constant in the scope of the thermo-mechanical model built. The setup operates dynamically under an argon atmosphere with a gas flow rate of $50 \mathrm{ml} / \mathrm{min}$ and a heating rate of $20 \mathrm{~K} / \mathrm{min}$. The heat flux principle considers a sample $(S)$ and a reference $(R)$ specimen, typically sapphire, subjected to a pre-determined temperature programme including heating and cooling cycles. The measurement process consists of three main stages, where the behavior of the empty calorimeter is initially determined as a base line $(B)$. This is followed by measurement of the reference material and finally measurement of the sample under analysis.

Specific heat capacity is defined in Eq. (6) together with the mathematical description of heat content of a material 


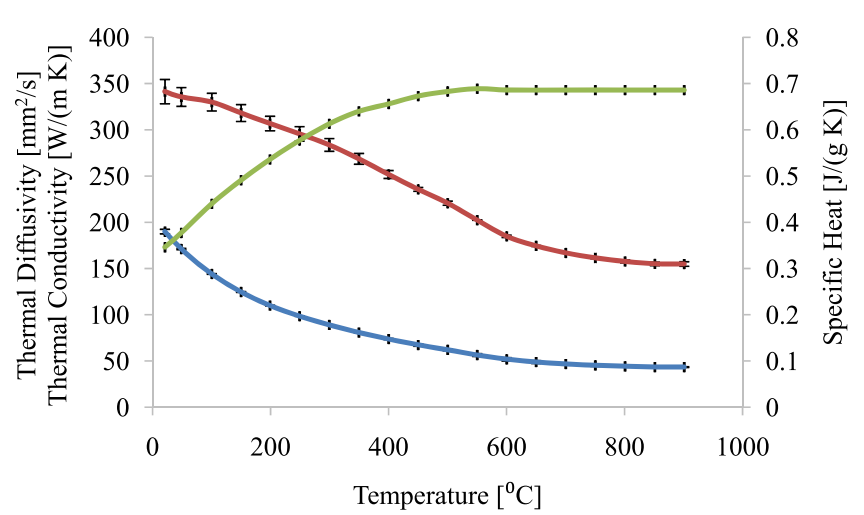

—Thermal Diffusivity — Thermal Conductivity — Specific Heat

FIG. 4. Plot of thermal diffusivity, thermal conductivity, and specific heat against temperature for $\mathrm{CuCD}$.

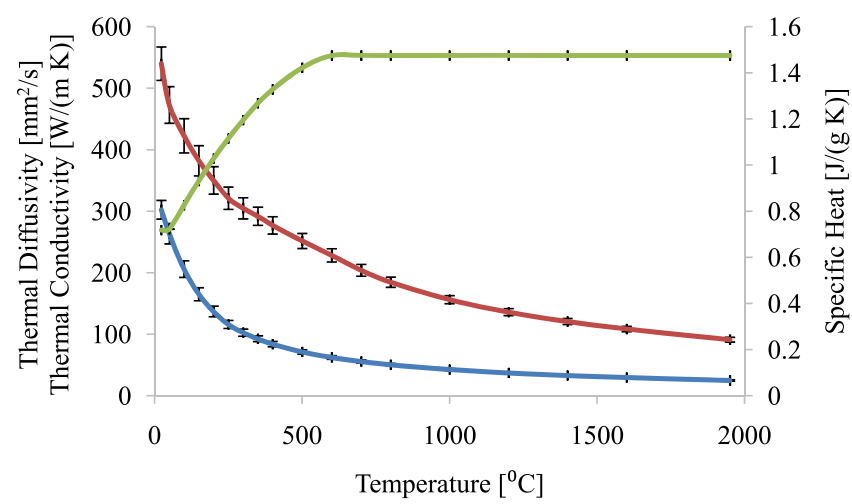

— Thermal Diffusivity — Thermal Conductivity — Specific Heat

FIG. 5. Plot of thermal diffusivity, thermal conductivity, and specific heat against temperature for MoGr along $(L)$.

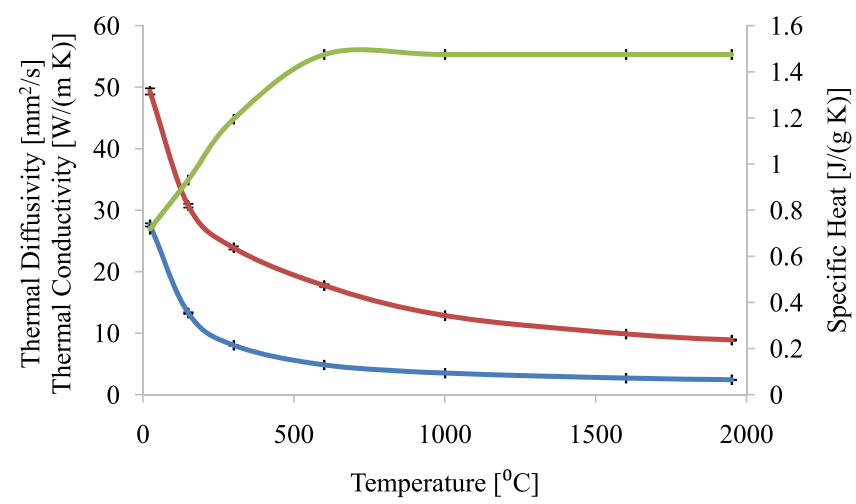

—Thermal Diffusivity — Thermal Conductivity — Specific Heat

FIG. 6. Plot of thermal diffusivity, thermal conductivity and specific heat against temperature for MoGr along $(T)$.

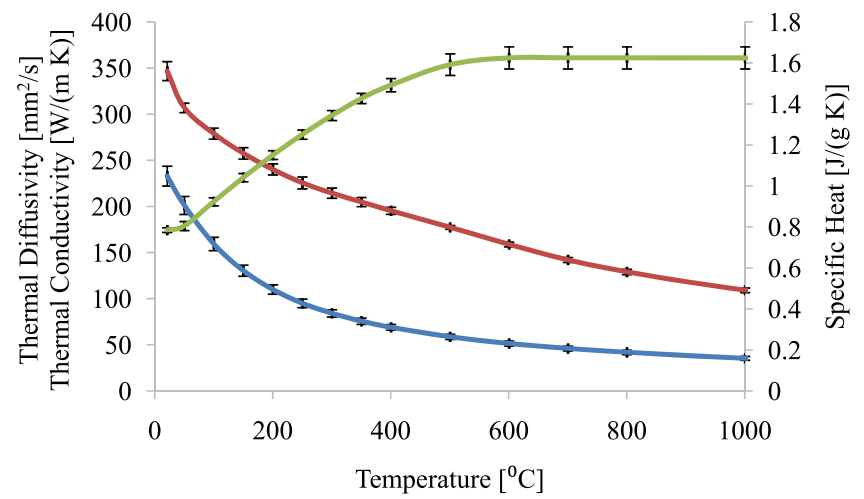

—Thermal Diffusivity — Thermal Conductivity — Specific Heat

FIG. 7. Plot of thermal diffusivity, thermal conductivity, and specific heat against temperature for CFC along $(L)$.

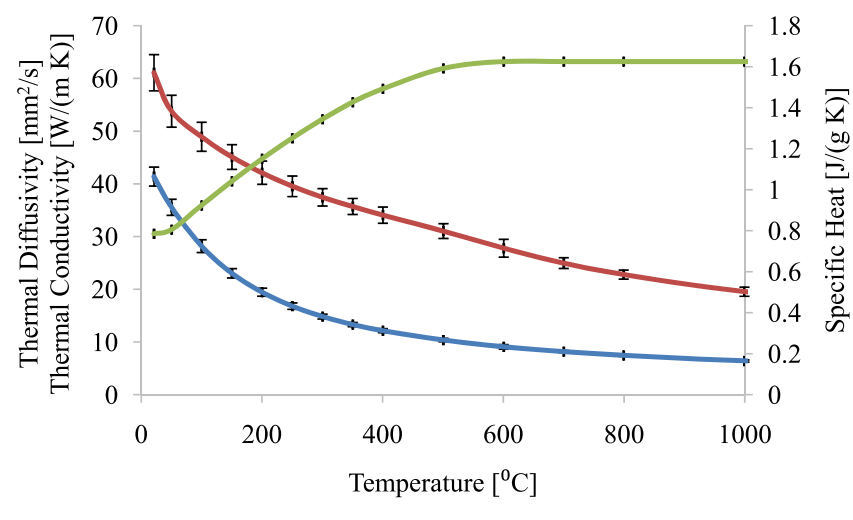

—Thermal Diffusivity — Thermal Conductivity — Specific Heat

FIG. 8. Plot of thermal diffusivity, thermal conductivity, and specific heat against temperature for $\mathrm{CFC}(T)$.

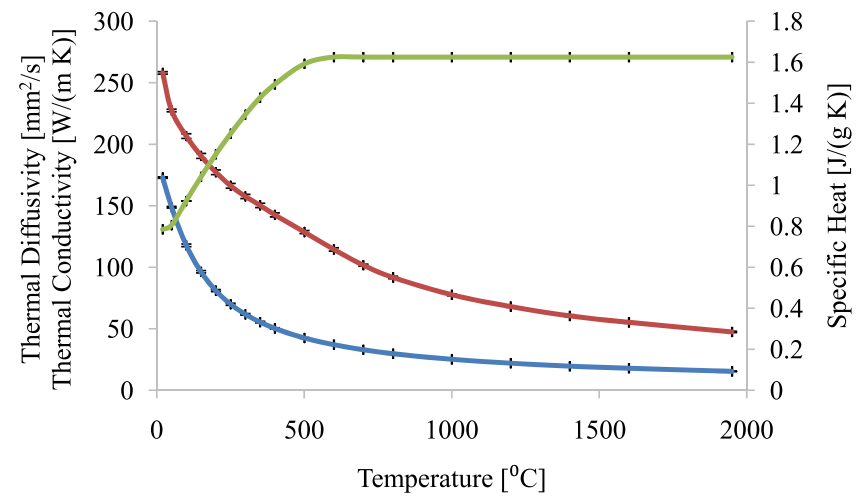

—Thermal Diffusivity — Thermal Conductivity — Specific Heat

FIG. 9. Plot of thermal diffusivity, thermal conductivity, and specific heat against temperature for CFC along $(P)$. 
TABLE II. Thermal properties for HRMT-23 jaw absorbers.

\begin{tabular}{lcccr}
\hline \hline Insert type & $\begin{array}{c}\text { Thermal diffusivity } \\
{\left[\mathrm{mm}^{2} / \mathrm{s}\right]}\end{array}$ & $\begin{array}{c}\text { Thermal conductivity } \\
{[\mathrm{W} /(\mathrm{m} \mathrm{K})]}\end{array}$ & $\begin{array}{c}\text { Specific heat } \\
{[\mathrm{J} /(\mathrm{g} \mathrm{K})]}\end{array}$ & $\begin{array}{c}\text { Coefficient of thermal } \\
\text { expansion }\left(\times 10^{-6}\right)\left[{ }^{\circ} \mathrm{K}^{-1}\right]\end{array}$ \\
\hline CuCD & 189.94 & 341.34 & 0.69 & 9.67 \\
MoGr $(L)$ & 302.32 & 539.88 & 1.48 & 1.48 \\
MoGr $(T)$ & 27.62 & 49.32 & 1.48 & 1.84 \\
CFC $(L)$ & 232.98 & 346.78 & 1.63 & -1.46 \\
CFC $(T)$ & 41.39 & 61.09 & 1.63 & 8.59 \\
CFC $(P)$ & 172.99 & 258.02 & 1.63 & -1.46 \\
\hline \hline
\end{tabular}

as a function of temperature, denoted in Eq. (7). Combining both equations leads to the derivation of the dynamic calorimeter calculation in Eq. (8), where $\rho$ represents specimen density, $V$ represents specimen volume, and DSC refers to the sensor signal, allowing specific heat $C_{p}$ calculation. The results of the LFA and DSC techniques are observed in Figs. 4-9, displaying mean plots as a result of repetitive testing to ensure statistical repeatability and control data scattering, with thermal parameters shown in Table II,

$$
\begin{gathered}
\Delta Q=C_{P} m \Delta T \\
Q(t)=\oint_{V} d V \quad \rho(\vec{x}, T) \int_{0}^{T(\vec{x}, T)} C_{P}(\vec{x}, T) d T \\
C_{p S}=\frac{D S C_{S}-D S C_{B}}{D S C_{R}-D S C_{B}} \frac{m_{R}}{m_{S}} C_{p R} .
\end{gathered}
$$

\section{Dilatometry}

Dilatometry (DIL) measures longitudinal expansion of a reference specimen, with a known coefficient of thermal expansion (CTE), and longitudinal expansion of an unknown specimen. The experimental setup consists of a high-temperature horizontal pushrod NETZSCH DIL $402 \mathrm{C}$ dilatometer operating within a thermal range of 22 to $2000{ }^{\circ} \mathrm{C}$, performing measurements in accordance with the ASTM E228-11 standard [22]. A cylindrical specimen is placed in a furnace containing a controlled helium environment with a gas flow rate of $50 \mathrm{ml} / \mathrm{min}$ and a heating rate of $2 \mathrm{~K} / \mathrm{min}$. The thermal testing ranges and incremental divisions are set at specific time intervals, with temperature recorded via a thermocouple adjacent to the specimen. During the heating cycle, the specimen expands and imparts a pressure proportional to the imparted displacement on the graphitic pushrod. The signal is transferred to a linear variable differential transformer system and converted into numerical strain. Both the connecting rod and the sample holder are exposed to the same atmospheric temperature as the specimen, with the final results being a summation of length variations of all the setup components. To cater for this, the equipment expansion behavior is carried out utilizing the reference material, followed by the specimen measurement.

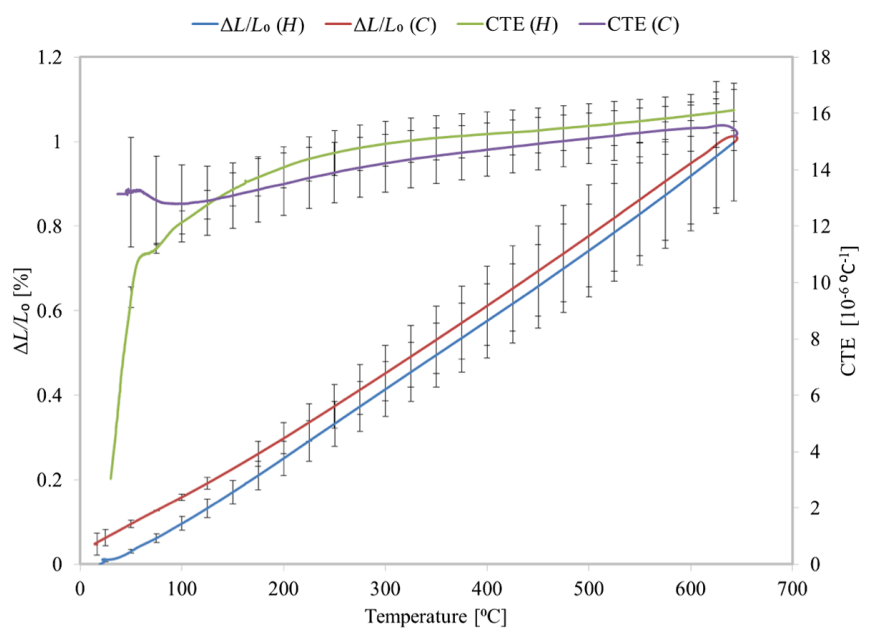

FIG. 10. Relative change in length and CTE against temperature with initial thermal reference of $49^{\circ} \mathrm{C}(H)$ and $50.095^{\circ} \mathrm{C}$ (C) for $\mathrm{CuCD}$.

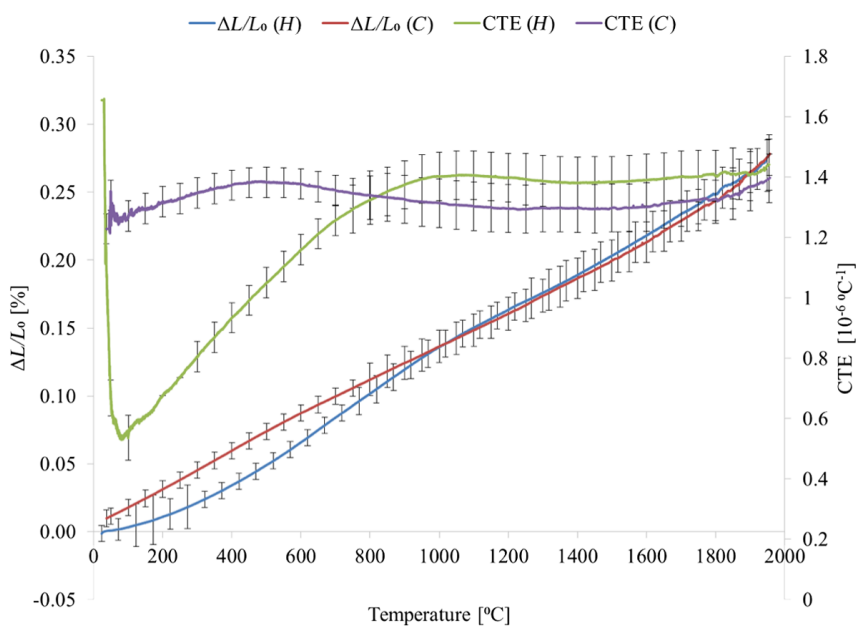

FIG. 11. Relative change in length and CTE against temperature with initial thermal reference of $100^{\circ} \mathrm{C}(H)$ and $41^{\circ} \mathrm{C}$ $(C)$ for MoGr along $(L)$. 


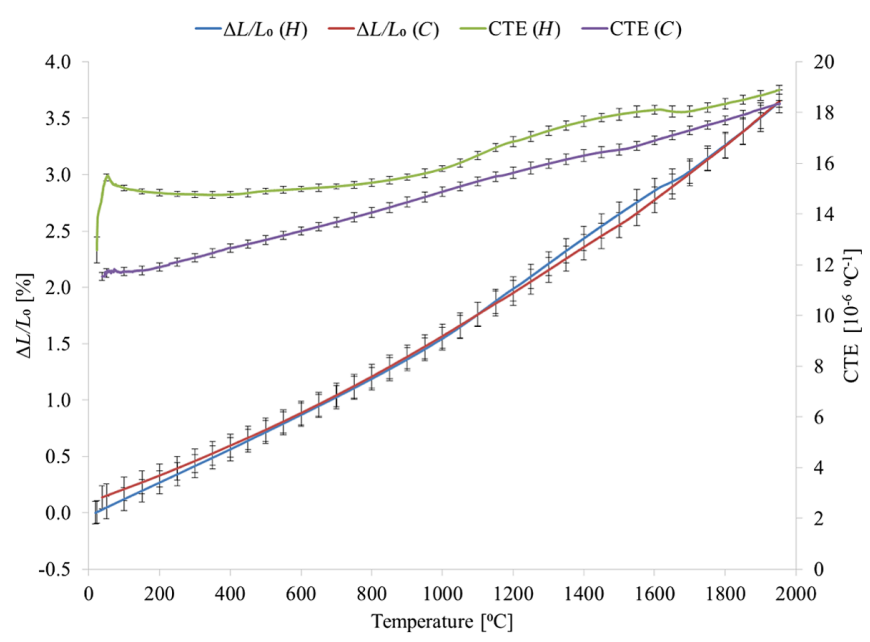

FIG. 12. Relative change in length and CTE against temperature with initial thermal reference of $48^{\circ} \mathrm{C}(H)$ and $50.1^{\circ} \mathrm{C}$ $(C)$ for MoGr along $(T)$.

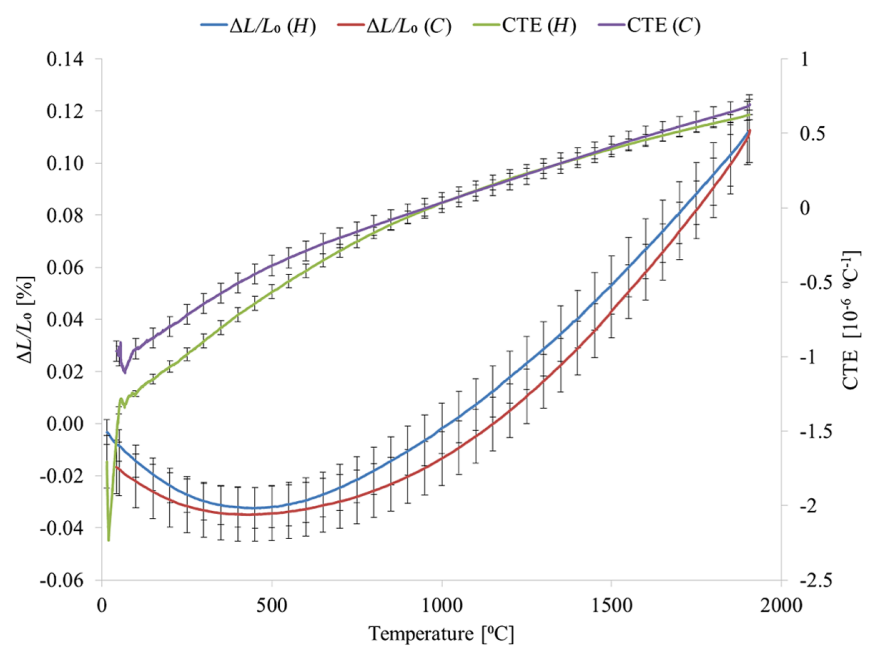

FIG. 13. Relative change in length and CTE against temperature with initial thermal reference of $42.7^{\circ} \mathrm{C}(H)$ and $50.03^{\circ} \mathrm{C}$ (C) for CFC along $(L)$.

The CTE for an unknown specimen material is defined by Eq. (9), where $\frac{\Delta L}{L_{0}}$ represents the change in specimen length corresponding to a specified change in temperature $\Delta T$. The results are depicted in Figs. 10-14, showing both the dimensional specimen length-temperature change and the calculated CTE against temperature during both heating $(H)$ and cooling $(C)$ cycles. Residual deformation is observed by the discrepancies at the heating start and cooldown end cycles. The CTE is obtained by using the plots of dimensional change against temperature for the cooling cycles from start to end points, with results shown in Table II,

$$
\mathrm{CTE}=\frac{1}{\Delta T}\left(\frac{\Delta L}{L_{0}}\right)_{S}
$$

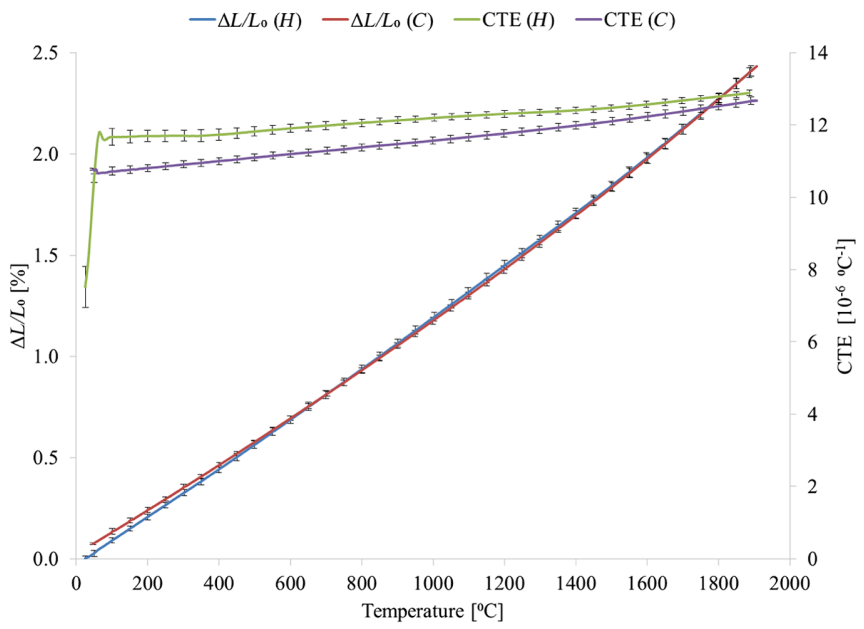

FIG. 14. Relative change in length and CTE against temperature with initial thermal reference of $100.1^{\circ} \mathrm{C}(H)$ and $42.9^{\circ} \mathrm{C}$ $(C)$ for CFC along $(T)$.

\section{STRUCTURAL CHARACTERIZATION}

Equation (10) is Hooke's law for an orthotropic material, where $\varepsilon$ is strain, $E$ is Young's modulus, $v$ is Poisson's ratio, $G$ is the shear modulus of elasticity, and $\sigma$ is stress: for all quantities the direction is denoted by the subscript. The strain and stress tensor are linked by the compliance matrix [S], which contains the following elastic constants: elastic modulus $(E)$, shear modulus $(G)$, and Poisson's ratio $(v)$ [23]. Parameters occurring along the main matrix diagonal are obtained via structural characterization testing, whereas the off-diagonal parameters may be obtained through relations with the main diagonal parameters. For an orthotropic case, Eq. (10) indicates nine independent elastic constants with five independent constants for a transversely isotropic case and three constants for an isotropic case, due to the introduction of symmetry planes. This is reflected in the parameters for a transversely isotropic case being identical along the $(L)$ and $(P)$ orientations, whereas for an isotropic case the parameters are identical along all the three orthogonal planes.

For the structural characterization campaign, four techniques target the elastic response for all material orientations over a low strain-rate range, at room temperature. These consist of flexural testing, compression testing, impact excitation testing (IET), and ultrasonic (US) testing,

$$
\left[\begin{array}{c}
\varepsilon_{X X} \\
\varepsilon_{Y Y} \\
\varepsilon_{Z Z} \\
2 \varepsilon_{Y Z} \\
2 \varepsilon_{Z X} \\
2 \varepsilon_{X Y}
\end{array}\right]=\left[\begin{array}{cccccc}
\frac{1}{E_{X}} & -\frac{v_{Y X}}{E_{Y}} & -\frac{v_{Z X}}{E_{Z}} & 0 & 0 & 0 \\
-\frac{v_{X Y}}{E_{X}} & \frac{1}{E_{Y}} & -\frac{v_{Z Y}}{E_{Z}} & 0 & 0 & 0 \\
-\frac{v_{X Z}}{E_{X}} & -\frac{v_{Y Z}}{E_{Y}} & \frac{1}{E_{Z}} & 0 & 0 & 0 \\
0 & 0 & 0 & \frac{1}{G_{Y Z}} & 0 & 0 \\
0 & 0 & 0 & 0 & \frac{1}{G_{Z X}} & 0 \\
0 & 0 & 0 & 0 & 0 & \frac{1}{G_{X Y}}
\end{array}\right]\left[\begin{array}{c}
\sigma_{X X} \\
\sigma_{Y Y} \\
\sigma_{Z Z} \\
\sigma_{Y Z} \\
\sigma_{Z X} \\
\sigma_{X Y}
\end{array}\right] .
$$




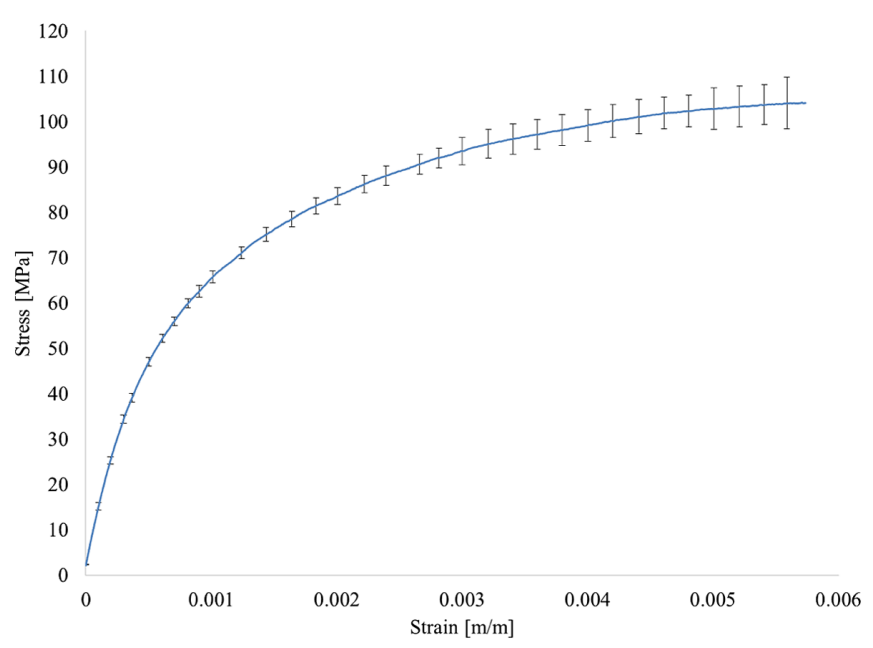

FIG. 15. Plot of flexural stress against strain for $\mathrm{CuCD}$.

\section{A. Flexural testing}

The elastoplastic response is given by the stress-strain response of a material tested under bending with a four point flexural setup, which consists of a universal Zwick Roell Z-100 machine with a maximum load and travel speed of $100 \mathrm{kN}$ and $5 \mathrm{~mm} / \mathrm{s}$, respectively, operating in accordance with the ASTM C1161-02c standard [24]. A strain gauge is attached to the lower part of a rectangular specimen, experiencing tensile effects, to record the stress variation at predefined loading steps until specimen failure. A load is applied at the midpoint of the loading member, imparting the load equally amongst both the upper loading pins until failure, ensuring that the center span is stressed in bending to avoid the presence of shear stresses. The results are depicted in Figs. 15-17 showing the incremental stress variation against strain, with elastic moduli results shown in Table III obtained in the initial linear regions of each respective plot.

\section{B. Compression testing}

Compression testing is a destructive test utilized to establish behavior of materials under the influence of a compressive force until failure. The experiment operates in accordance with the ASTM C695-91 standard [25]. A short cylindrical specimen with attached rosette strain gauges is vertically set within a universal Zwick Roell Z-100 testing machine, set up with two parallel supporting contacting steel blocks. A load is gradually imparted at preset steps to the loading member with a rate of $2 \mathrm{~mm} / \mathrm{s}$, while the corresponding load against strain data is recorded via strain gauges until specimen rupture. The results are depicted in Figs. 18-20, with elastic moduli results tabulated in Table III, obtained in the initial linear regions of each respective plot, reflecting the isotropic, transversely isotropic, and orthotropic nature of the $\mathrm{CuCD}, \mathrm{MoGr}$, and $\mathrm{CFC}$ composite, respectively.

\section{Impact excitation testing}

IET characterization aims at analyzing the vibration behavior of specimens, carried out in accordance with the ASTM C1259-01 [26] and ASTM C747-93 [27] standards. The vibrational modes under analysis include both the flexural and torsional frequencies operating at the

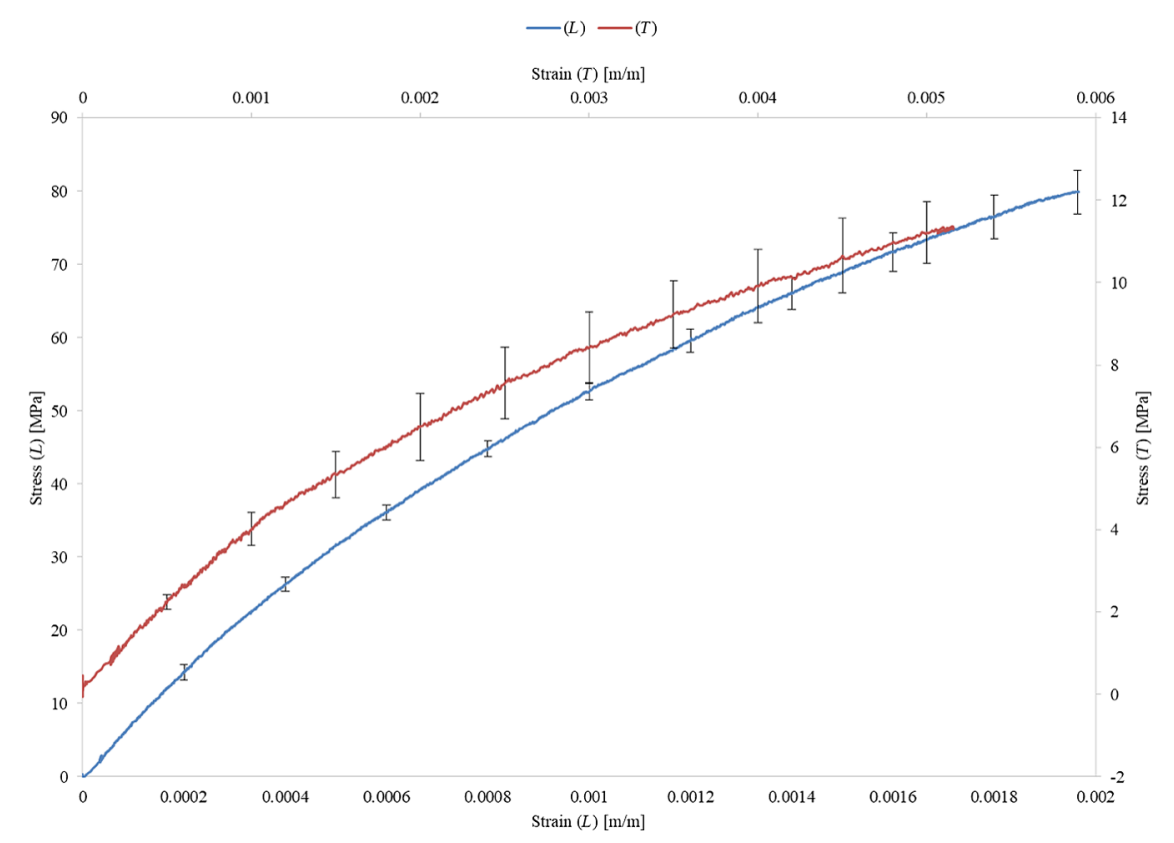

FIG. 16. Plot of flexural stress against strain for MoGr. Longitudinal data and relevant axes marked with $(L)$. Transverse data and relevant axes marked with $(T)$. 


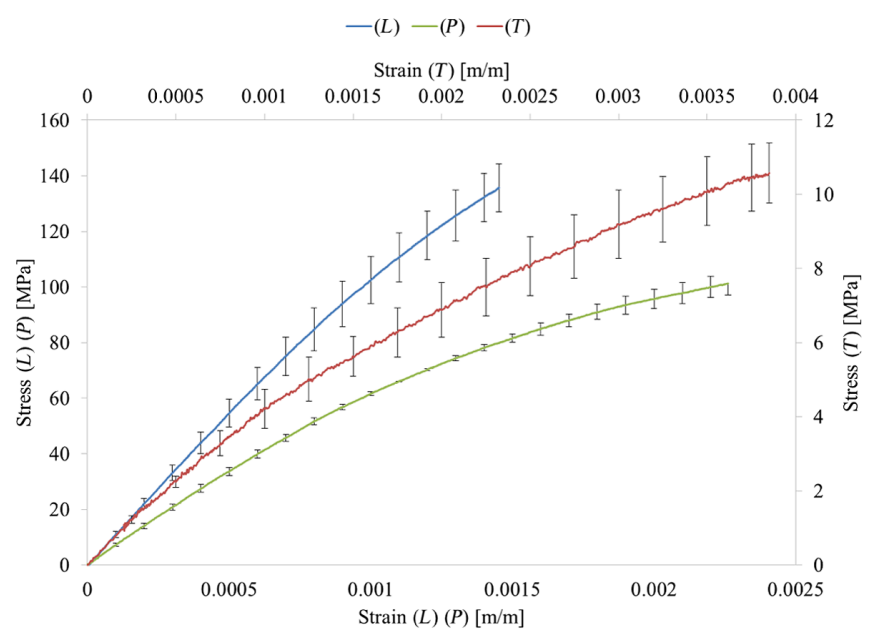

FIG. 17. Plot of flexural stress against strain for CFC. Longitudinal data and relevant axes marked with $(L)$. Transverse data and relevant axes marked with $(T)$. Perpendicular data and relevant axes marked $(P)$.

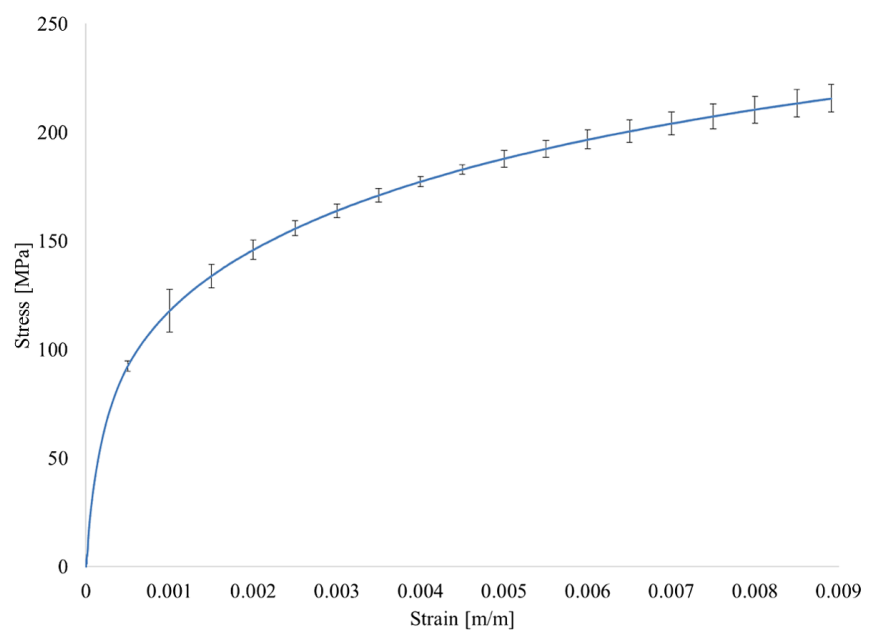

FIG. 18. Plot of compressive stress against strain for $\mathrm{CuCD}$.

first vibrational modes, denoted as $f_{f}$ and $f_{t}$, respectively. Rectangular specimens with a high length-to-thickness ratio are subjected to minute strains via a light strike with an impulse tool. The specimen supports, impulse locations, and signal pick-up points are selected to induce and measure selective vibrational modes. The resulting signal is picked up through a noncontacting microphone transducer and converted into an electrical signal. The

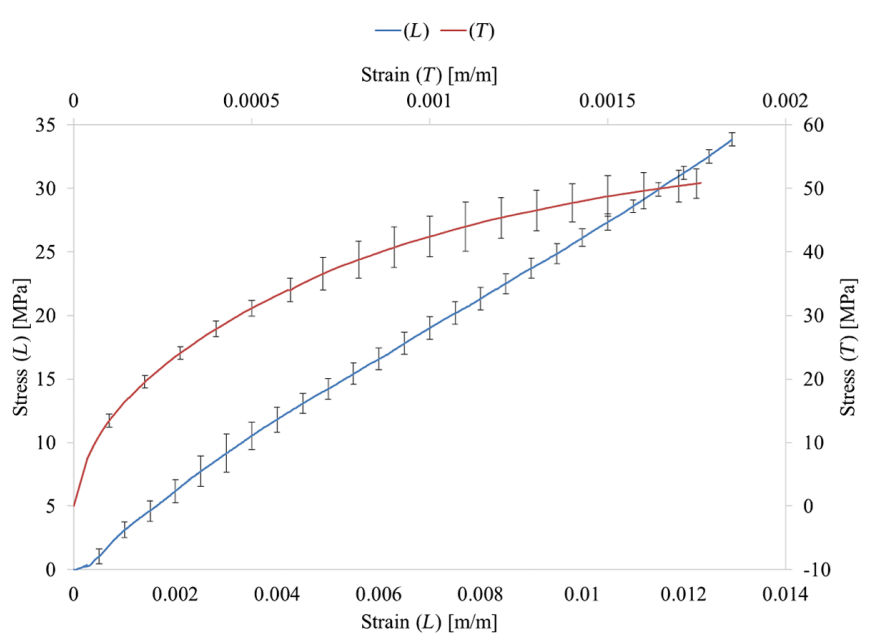

FIG. 19. Plot of compressive stress against strain for MoGr. Longitudinal data and relevant axes marked with $(L)$. Transverse data and relevant axes marked with $(T)$.

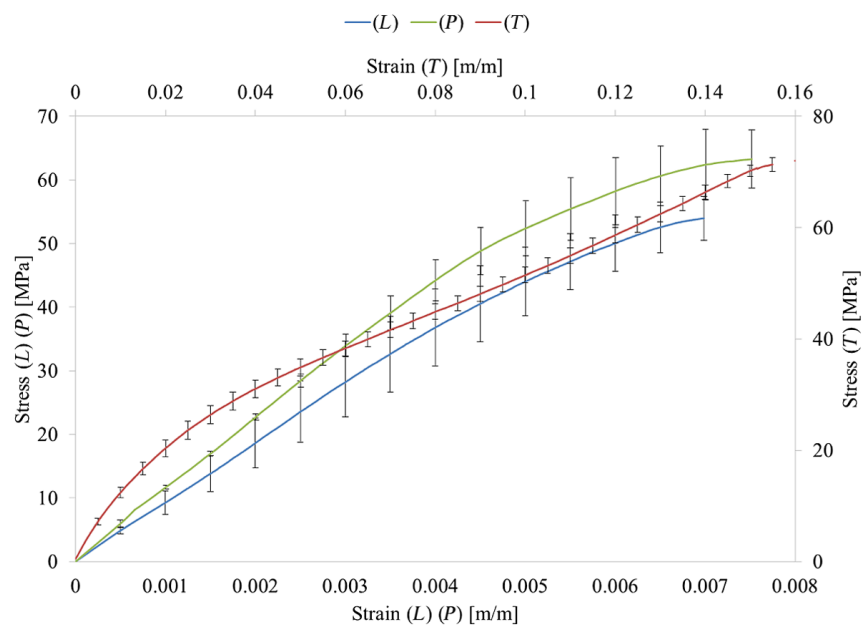

FIG. 20. Plot of compressive stress against strain for CFC. Longitudinal data and relevant axes marked with $(L)$. Transverse data and relevant axes marked with $(T)$. Perpendicular data and relevant axes marked $(P)$.

signal is then amplified, converted into a quantifiable numerical frequency, and read out to a computer-aided postprocessing software package. This leads to the determination of dynamic elastic properties at or near the origin of the stress-strain curve, with minimum possibility of fracture.

TABLE III. Flexural and compressive elastic moduli [GPa] along the respective orientations.

\begin{tabular}{|c|c|c|c|c|c|c|}
\hline \multirow[b]{2}{*}{ Insert type } & \multicolumn{2}{|c|}{ Longitudinal $(L)$} & \multicolumn{2}{|c|}{ Transverse $(T)$} & \multicolumn{2}{|c|}{ Perpendicular $(P)$} \\
\hline & Flexural & Compressive & Flexural & Compressive & Flexural & Compressive \\
\hline $\mathrm{CuCD}$ & 155.76 & 306.37 & 155.76 & 306.37 & 155.76 & 306.37 \\
\hline $\mathrm{MoGr}$ & 73.61 & 2.99 & 3.82 & 176.39 & 73.61 & 2.99 \\
\hline CFC & 115.61 & 1.28 & 4.78 & 4.58 & 68.78 & 6.88 \\
\hline
\end{tabular}


TABLE IV. Mean resonance testing flexural frequency $\mathrm{f}_{f}[\mathrm{~Hz}]$, torsional frequency $\mathrm{f}_{t}[\mathrm{~Hz}]$, and elastic parameters, including elastic modulus $E[\mathrm{GPa}]$, shear modulus $G[\mathrm{GPa}]$, and Poisson's ratio $v$, for $\mathrm{CuCD}$, MoGr, and CFC along $(L),(T)$, and $(P)$.

\begin{tabular}{|c|c|c|c|c|c|c|}
\hline Insert type & & $\mathrm{f}_{f}[\mathrm{kHz}]$ & $\mathrm{f}_{f}[\mathrm{kHz}]$ & $E$ [GPa] & $G$ [GPa] & $v$ \\
\hline \multirow[t]{2}{*}{$\mathrm{CuCD}$} & mean & 34.63 & 49.56 & 125.00 & 66.90 & -0.066 \\
\hline & SD & 0.15 & 0.30 & 0.28 & 0.92 & 4.98 \\
\hline MoGr & mean & 8.40 & 15.68 & 62.78 & 14.38 & 1.18 \\
\hline$(L)$ & SD & 1.14 & 0.79 & 1.78 & 1.61 & 3.49 \\
\hline MoGr & mean & 10.58 & 17.10 & 6.69 & 4.58 & -0.27 \\
\hline$(T)$ & $\mathrm{SD}$ & 1.58 & 0.58 & 1.01 & 1.29 & 4.72 \\
\hline $\mathrm{CFC}$ & mean & 11.88 & 15.93 & 89.18 & 9.28 & 3.81 \\
\hline$(L)$ & SD & 0.129 & 0.75 & 1.44 & 1.72 & 2.38 \\
\hline $\mathrm{CFC}$ & mean & 8.33 & 15.51 & 3.52 & 2.14 & -0.18 \\
\hline$(T)$ & SD & 0.88 & 0.74 & 2.75 & 2.42 & 4.88 \\
\hline $\mathrm{CFC}$ & mean & 9.22 & 14.26 & 52.15 & 7.16 & 2.64 \\
\hline$(P)$ & $\mathrm{SD}$ & 1.19 & 1.26 & 2.38 & 3.12 & 4.65 \\
\hline
\end{tabular}

For dynamic elastic moduli, the empirical relation represented in Eq. (11) is applied in accordance with the analytical resonance theories of Timoshenko, Goens, and Pickett [26]. This is created such that numerical comparisons of these combined theories, up to four significant figures, are justified, assuming an arbitrary Poisson's ratio of 0.292 as specified in the relevant standard [28,29]. Equation (11) is considered reliable through the inclusion of a shape correction factor $\left(T_{l}\right)$, able to account for the finite bar thickness and Poisson's ratio variations [28]. Pickett identified a general empirical relation for shear moduli obtained from the first torsional frequency by using two correction factors $\mathrm{A}_{o}$ and $\mathrm{B}_{o}$, as shown in Eq. (12) [30]. It is assumed that the relationship between resonance frequencies and dynamic moduli is valid for a material that is homogeneous, elastic, and isotropic, as per the relevant standards. Considering this, it is implied that an improved Poisson's ratio value is obtained by Eq. (13),

$$
\begin{gathered}
E=0.9465\left(m \frac{f_{f}^{2}}{B}\right)\left(\frac{L^{3}}{T^{3}}\right)\left[1.000+6.585\left(\frac{T}{L}\right)^{2}\right] T_{l}, \\
G=\left(\frac{4 L m f_{t}^{2}}{B T}\right)\left(\frac{B_{o}}{1+A_{o}}\right), \\
v=\frac{E}{2 G}-1 .
\end{gathered}
$$

The frequency and corresponding elastic parametric results are shown in Table IV together with the accompanying standard deviation (SD).

For $\mathrm{CuCD}$, a small SD results for both $E$ and $G$, with a contrasting significantly high SD for $v$, reflecting a lack of accuracy amongst sample measurements. A similar pattern is observed for both MoGr and CFC along all orientations, where Poisson's ratios have the highest SD. Typically
Poisson's ratios vary within a range of $0-0.5$, where excessively large or negative values beyond this range are not considered physically possible. Table IV indicates that both negative and excessively large Poisson's ratios result from experimentally obtained elastic and shear moduli. Lauwagie et al. [31] noted similar results when carrying out experimental resonance beam tests on commercially available orthotropic 6082 aluminum alloy and 304 stainless steel. The results from resonance beam testing failed to accurately model the directional variation of both $E$ and $G$, leading to an inaccurate Poisson's ratio identification when compared to other testing techniques. This is due to the fact that the relation depicted in Eq. (13) is based on a fully isotropic material model, which is not always the case.

\section{Ultrasonic testing}

Measuring ultrasonic velocities in materials with conventional ultrasonic pulse echo flaw detection equipment is utilized to aid in optimization of elastic parameters. The setup consists of an ultrasonic instrument, Krautkramer USN60, and straight beam longitudinal wave transducers, GE MSW-QC2.25 ( $\varnothing 0.5 ”, 2.25 \mathrm{MHz})$ and GE Alpha 2 $(\varnothing 5 \mathrm{~mm}, 10 \mathrm{MHz})$, used in a contact mode with a plexiglas delay line and water as couplant. Ultrasonic velocity measurements are carried out by comparing transmission

TABLE V. Mean speed of sound for respective material specimens.

\begin{tabular}{lccc}
\hline \hline Insert type & Orientation & $\begin{array}{c}\text { Mean sound } \\
\text { speed }[\mathrm{m} / \mathrm{s}]\end{array}$ & $\begin{array}{c}\text { Standard deviation } \\
{[ \pm \mathrm{m} / \mathrm{s}]}\end{array}$ \\
\hline CuCD & $/$ & 6079 & 1.59 \\
& $(L)$ & 1475 & 2.09 \\
MoGr & $(T)$ & 6510 & 1.26 \\
\hline \hline
\end{tabular}


times of a longitudinal wave pulse through a specimen to its travel path along the entire specimen span [32].

Results show that homogeneity is present along and between each specimen, with the final sound speeds tabulated in Table V. No results are attainable for CFC due to the difficulties encountered during experimentation, most likely occurring due to the high degree of absorption of the specimens. For MoGr, results show a difference between the sound speeds along $(L)$ and $(T)$, possibly due to variations between stacking of the internal graphitic layers. A higher sound speed is noted along $(T)$, possibly due to the orientation reflecting the material pressing direction during manufacture, leading to a densely packed graphitic structure along the specific direction, allowing for easier sound wave transfer.

\section{STRUCTURAL CHARACTERIZATION OPTIMIZATION}

\section{A. Optimization technique}

The method reported in Sec. III C fails to determine the elastic constants of the orthotropic materials because the relationships used for the elastic constants are relevant to isotropic cases. Therefore, in the most general sense, the problem must be solved numerically, with a modal analysis targeting the first flexural and torsional frequencies, which are also measured experimentally as a function of the parameters of the compliance matrix. This section deals with determination of the elastic constants, including elastic moduli, shear moduli, and Poisson's ratios, for the orthotropic materials. The calculations are done by an educated guess of the compliance matrix parameters and the calculation of the resulting numerical frequencies together with an optimization procedure carried out in ANSYS, whose scope is to minimize the error between numerical and experimental frequencies by manipulation of the matrix constants.

The initial stage of optimization is similar to the approach adopted by Lauwagie et al. [31], where IET and tensile tests are used together with a mixed numerical experimental technique (MNET). The principle of the MNET is to compare experimentally measured frequencies with numerically computed frequencies by implementing a mock numerical model of the experimental setup. The properties obtained are iteratively refined until an acceptable precision limit is reached. This technique is also successfully adopted by Hosten [33] for polyether etherketone-carbon fiber composites and Grediac and Paris [34] for anisotropic metal plates. Pronounced variations are present for Poisson's ratios for all cases, because they were computed from fully isotropic behavioral assumptions, considered during IET. To cater for this, an alteration is applied using the US technique as proposed by Kohlhauser et al. [35], mostly applicable for anisotropic materials. Combining both methodologies, including results from the characterization campaign, an optimization chain is

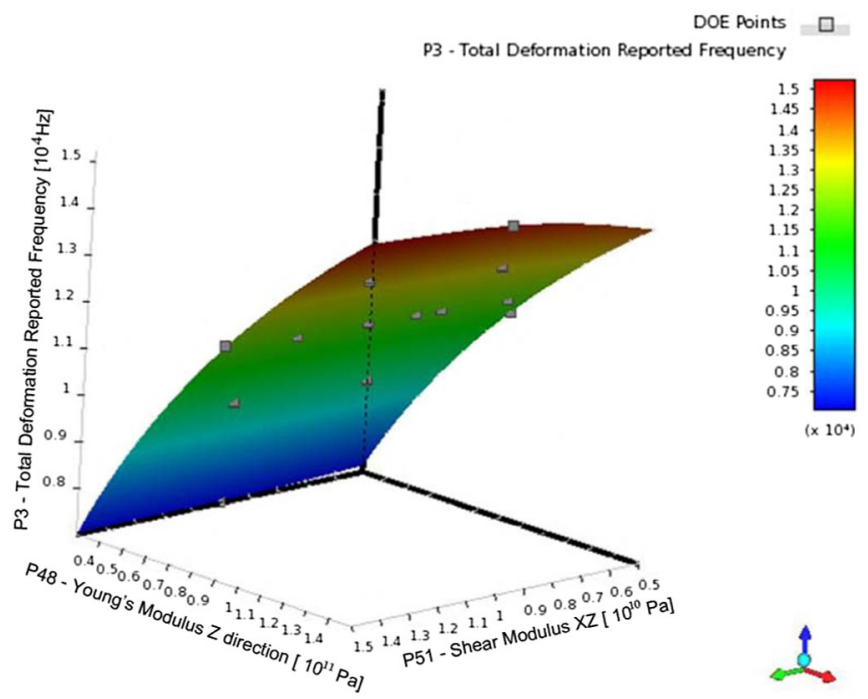

FIG. 21. 3D response surface for $E_{z}[\mathrm{~Pa}]$ against $G_{x z}[\mathrm{~Pa}]$ against flexural frequency $[\mathrm{Hz}]$.

developed. This optimization chain is based on the use of the experimental data and the first flexural and torsional frequencies as the target of the numerical analysis.

Educated values of the elastic constants are the starting point of the numerical analysis; the set of constants is used by ANSYS to determine the compliance matrix, solving then the eigen-frequency problem and comparing the results with the target experimental frequency. The optimization module within ANSYS then automatically updates the elastic constants values and relaunches the analysis, with the objective of minimizing the difference between simulated and target values, until the difference is reduced to $1 \%$ or less. The geometric model replicates both the geometry and the free-free boundary conditions experienced by specimens during structural IET and US characterization. Because of inaccurate and nonphysical Poisson's ratios reported in Sec. III, an educated guess of 0.1 was used as the initial Poisson's ratio value, to be later confirmed by US measurements.

The optimization algorithm used to minimize the difference between numerical and experimental frequencies starts with a design of experiment. The initial step involves identification of the design parameters under experimentation, including identification of both the input and output parameters of interest. The numerical ranges within which the input parameters may lie are selected based on physical considerations, and, in particular, by imposing that no constant can be negative or higher than $1000 \mathrm{MPa}$, while Poisson's ratios are constrained between 0 and 0.5. From the available combinations, an adequate amount of design points are automatically selected from the maximum and minimum boundaries of the output parameters, creating a design space within which optimization occurs. The design points are mapped to create a response surface, graphically depicting the relation between any desired inputs and 


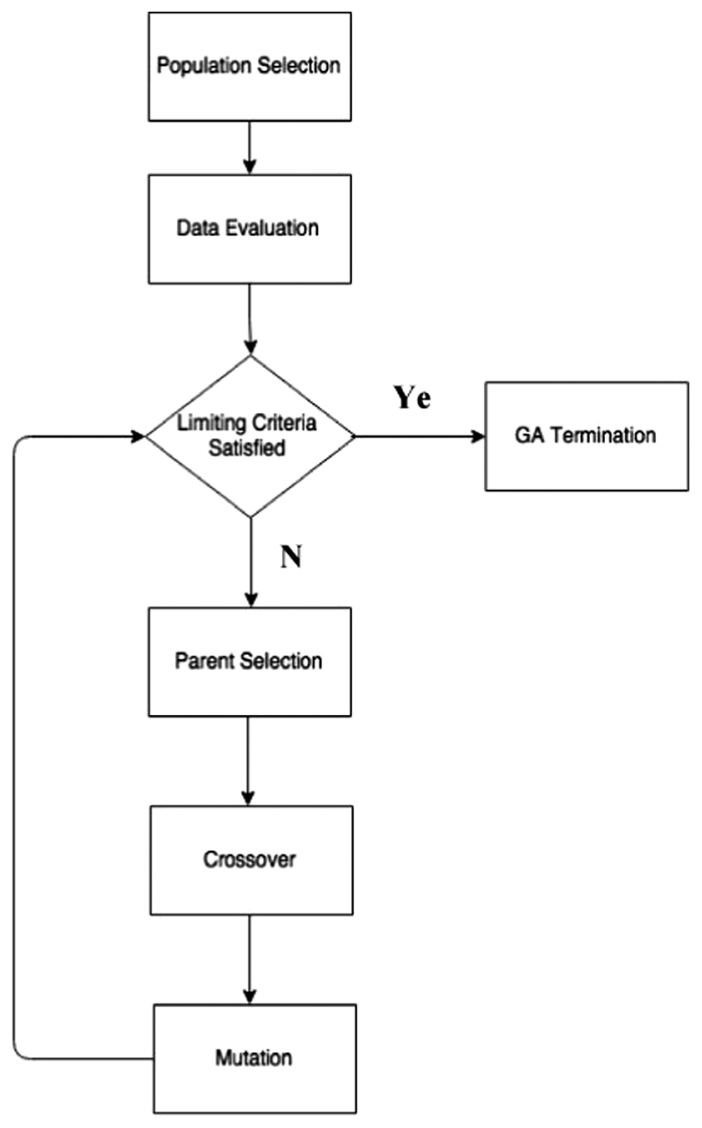

FIG. 22. Genetic algorithm approach.

outputs, as shown in Fig. 21. To carry out the optimization, specific objectives and constraints are set for output resonant frequencies. The targeted objectives set are the experimental resonant frequencies. For the target constraints, resonant frequencies are allowed to vary within a maximal frequency range set lower than the standard deviation results identified in Table IV. These constraints allow the optimization technique to provide results that exhibit lower deviation than those obtained experimentally. Additional constraints are introduced through parametric relationships, reflecting the inherent characteristic material behavior. For example, for MoGr both Young's and shear moduli are considered equal along the plane of isotropy.

The multiobjective genetic algorithm was used. This goal-driven optimization technique represents a class of algorithms that simulates the process of natural evolution via crossover and mutation techniques, specifically beneficial for multiple conflicting objectives, allowing for flexible problem formulation and availability of continuous input parameters, as shown in Fig. 22 [36].

Once the genetic algorithm is successful, a combination of final candidate sets are given, out of which the best results are selected. Using a multiobjective form of optimization does not provide a single solution for any input parameters but rather gives a set of possible solutions.

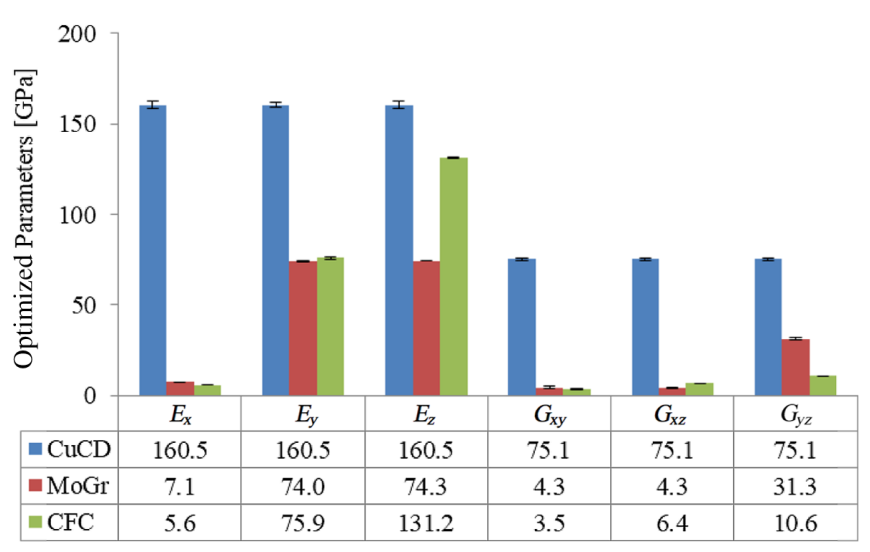

FIG. 23. Optimized elastic and shear moduli.

However, this technique is still desirable for problems involving multiple conflicting objectives and highly complex design spaces due to its ability to handle multiple goals, the flexible problem formulation, and the availability of continuous input parameters.

The general material stress-strain relationship is portrayed in terms of compliance $[S]$ and stiffness $[C]$ matrices, which is its inverse, Eqs. (14) and (15), respectively. This general case is applicable for orthotropic CFC, with symmetry along the 11-22 plane considered for $\mathrm{MoGr}$ and identical parameters along all orientations for $\mathrm{CuCD}$. The optimized elastic and shear moduli obtained from numerical modal analysis are inserted into the compliance matrix to define the constitutive material law. For a general orthotropic case, equations are available that relate ultrasonic sound velocities with compliance tensors along the relative directions [37]. This approach is adopted by Hosten et al. [33] where the product of stiffness and compliance tensors is used to numerically solve for off-diagonal stiffness tensor components. Knowing that the compliance tensors are the inverse of the stiffness matrix, Poisson's ratios are obtained utilizing both $[S]$ and $[C]$ matrices while ensuring that the variation does not exceed an acceptable limit of $1 \%$,

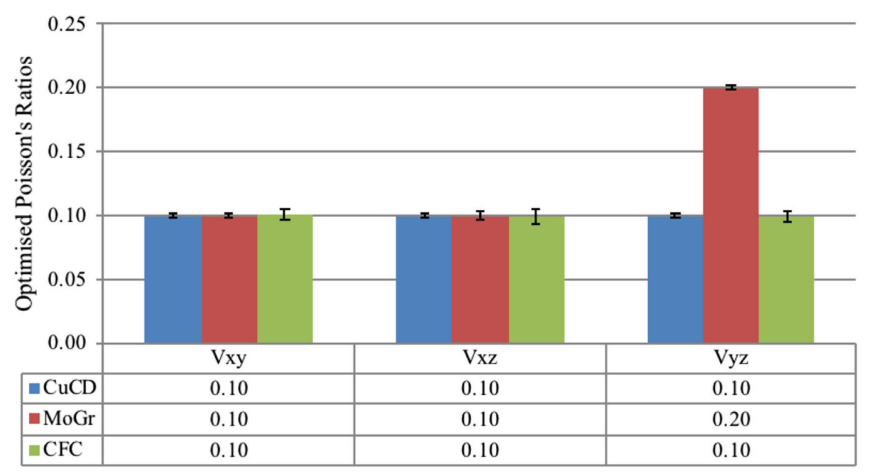

FIG. 24. Optimized elastic Poisson's ratios. 
TABLE VI. Figures of merit for current materials adopted in LHC collimators (CFC and tungsten alloy), requirements for HL-LHC, and figures for the materials under investigation.

\begin{tabular}{lcccccc}
\hline \hline & $\begin{array}{c}\text { Primary/secondary } \\
\text { HL-LHC collimator } \\
\text { minimum target }\end{array}$ & $\begin{array}{c}\text { Tertiary HL-LHC } \\
\text { collimator minimum } \\
\text { target }\end{array}$ & $\begin{array}{c}\text { Carbon- } \\
\text { carbon }\end{array}$ & $\begin{array}{c}\text { Tungsten } \\
\text { heavy } \\
\text { alloy }\end{array}$ & MoGr & CuCD \\
\hline TRI & 200 & 5 & $800-1200$ & 0.5 & 230 & 8.5 \\
TSI & 40 & 0.1 & 45 & 0.14 & 56 & 5 \\
RFI & 1 & 2.5 & 0.38 & 2.9 & 1 & 3.5 \\
\hline \hline
\end{tabular}

$$
[S]=\left[\begin{array}{cccccc}
\frac{1}{E_{Z}} & -\frac{v_{Y Z}}{E_{Y}} & -\frac{v_{X Z}}{E_{X}} & 0 & 0 & 0 \\
-\frac{v_{Z Y}}{E_{Z}} & \frac{1}{E_{Y}} & -\frac{v_{X Y}}{E_{X}} & 0 & 0 & 0 \\
-\frac{v_{Z X}}{E_{Z}} & -\frac{v_{Y X}}{E_{Y}} & \frac{1}{E_{X}} & 0 & 0 & 0 \\
0 & 0 & 0 & \frac{1}{G_{Y X}} & 0 & 0 \\
0 & 0 & 0 & 0 & \frac{1}{G_{X Z}} & 0 \\
0 & 0 & 0 & 0 & 0 & \frac{1}{G_{Z Y}}
\end{array}\right],
$$

$$
[C]=\left[\begin{array}{cccccc}
C_{11} & C_{12} & C_{13} & 0 & 0 & 0 \\
C_{12} & C_{22} & C_{23} & 0 & 0 & 0 \\
C_{13} & C_{23} & C_{33} & 0 & 0 & 0 \\
0 & 0 & 0 & C_{44} & 0 & 0 \\
0 & 0 & 0 & 0 & C_{55} & 0 \\
0 & 0 & 0 & 0 & 0 & C_{66}
\end{array}\right]
$$

\section{B. Results and evaluation}

The optimized elastic parameter results displayed in Figs. 23 and 24 indicate that $\mathrm{CuCD}$ has the highest elastic and shear moduli, reflecting significant stiffness when compared to both MoGr and CFC, which exhibit rather similar elastic behavior, possibly due to the similar microstructural graphitic nature. Figure 24 shows that Poisson's ratios are rather similar for each material along each direction and lie within a physically acceptable range of $0-0.5$, except for MoGr displaying a slight increase for $v_{y z}$.

\section{CONCLUSIONS}

In modern high-energy particle beam accelerators, accidental beam deviations are frequently experienced, imparting rapid internal heating and, in turn, inducing a dynamic response. The LHC accelerator allows for highenergy circulating beams, necessitating adequate machine protection devices during operation, such as collimators. Collimation systems may experience significant particlematter interactions with the highest energy deposition imparted onto absorbent materials. Understanding the thermophysical behavior of absorbers under such circumstances is critical to assist with the development of representative material models.

The thermostructural characterization campaign carried out within CERN research facilities for collimator jaw absorbers provides an overview of the three composite insert types along all orientations. Initial assumptions, focused on isotropic material response, were considered during the initial phases of the characterization. The need to obtain the full elastic parameters for transversely isotropic and orthotropic materials from the initial structural campaign data leads to the computation of the parameters by means of a combined Finite Element Method model and optimization procedure. Combining both experimental and numerical approaches a MNET is created. This approach indicates similarity of frequency mode results with impact excitation characterization results, reflecting a stable and robust
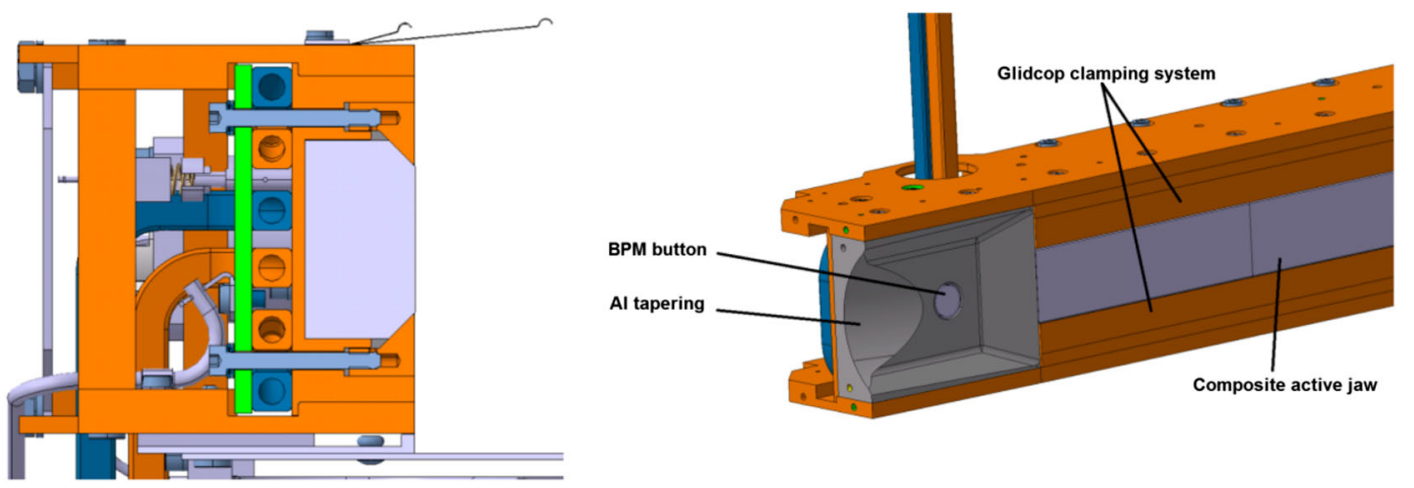

FIG. 25. New collimator modular design of collimator jaw [38]. 
approach and providing optimization of experimentally determined elastic properties. The optimized elastic parametric results indicate that $\mathrm{CuCD}$ is the stiffest composite, followed by CFC and finally MoGr. While this is an important result, it is clearly one of the many parameters to consider in determining the best material for the application. The figures of merit described earlier are reproduced in Table VI, with the inclusion of the values obtained from the data presented in this work. This shows how the data presented here aid in the selection of the most suitable material.

The results of this study confirm that MoGr satisfies the requirements for HL-LHC primary and secondary collimators, while CuCD meets the HL-LHC targets for tertiary collimators. The two materials are proposed for replacement of CFC and W-alloy, respectively. This has an impact on the design of the collimator jaw: MoGr, differently from $\mathrm{CFC}$, cannot be produced as a monolithic structure $1 \mathrm{~m}$ long, and $\mathrm{CuCD}$ cannot be threaded and screwed to the Glidcop support, as was done in the case of the W-alloy. A new modular design, Fig. 25, universal to primary, secondary, and tertiary collimator jaws, was studied, allowing to change the function of the collimator by simply replacing the absorber blocks in the housing [38]. The jaw can host $\mathrm{CuCD}$ or MoGr blocks interchangeably.

\section{ACKNOWLEDGMENTS}

This research has been supported through the FP7 EuCARD-2 "Enhanced European Coordination for Accelerator Research \& Development" which is co-funded by the partners and the European Commission under capacities of the 7th Framework Program, Grant Agreement No. 312453. The financial support of CERN and the University of Malta is also acknowledged. Moreover, the authors would like to acknowledge the contributions of the various teams involved: HiRadMat, EN-MME-EDM, EN-MME-EDS, and EN-MME-MM team.

[1] O. S. Brüning et al., CERN Technical Report No. CERN2004-003-V-1, 2004.

[2] A. Bejar and L. Rossi, CERN Technical Report No. CERN-ACC-2015-0140, 2015.

[3] L. Evans and J. Gareyte, CERN Technical Report No. CERN-SPS-82-8-DI-MST, 1982.

[4] A. Piwinski, in Proceedings of the 9th International Conference on High Energy Accelerators, Stanford, CA, pp. 405-409, 1974, http://inspirehep.net/record/964055.

[5] T. Pieloni,, D. Banfi, and J. Barranco Garcia, CERN Report No. CERN-ACC-NOTE-2017-0035, 2017

[6] A. Valishev, D. Shatilov, T. Pieloni, D. Banfi, and J. Barranco, in Proceedings of the 25th Particle Accelerator Conference, PAC-2013, Pasadena, CA, 2013 (IEEE, New York, 2013).

[7] M. Crouch, T. Pieloni, R. B. Appleby, D. Banfi, J. Barranco Garcia, R. Bruce, X. Buffat, B. D. Muratori, M. Pojer,
B. Salvachua, C. Tambasco, and G. Trad, Proceedings of IPAC2016, Busan, Korea, 2016, http://accelconf.web.cern .ch/AccelConf/ipac2016/.

[8] A. Bertarelli et al., in The 54th ICFA Advanced Beam Dynamics Workshop on High-Intensity, High Brightness and High Power Hadron Beams, http://accelconf.web.cern .ch/AccelConf/HB2014/talks/tho4ab03_talk.pdf.

[9] C. Bracco, Ph.D. dissertation, Ecole Polytechnique Federale de Lausanne, Lausanne, France, 2009.

[10] E. Quaranta, A. Bertarelli, R. Bruce, F. Carra, F. Cerutti, A. Lechner, S. Redaelli, E. Skordis, and P. Gradassi, Modeling of beam-induced damage of the LHC tertiary collimators, Phys. Rev. Accel. Beams 20, 091002 (2017).

[11] A. Bertarelli, F. Carra, N. Mariani, and S. Bizzaro, in Proceedings Tungsten, Refractory and Hardmetals Conference, Orlando, 2014, http://www.ebooksstandard .com/advances-in-tungsten-refractory-amp;-hardmaterialsix?manufacturer_id=123\&sort=pd.name\&order=ASC\& page $=2$.

[12] F. Carra, Ph.D. thesis, Politecnico di Torino, 2017.

[13] A. Bertarelli, in Proceedings of the Joint International Accelerator School: Beam Loss and Accelerator Protection, Newport Beach, 2014, edited by R. Schmidt, (CERN, Geneva, 2016).

[14] I. Efthymiopoulos et al. in Proceedings of the 2nd International Particle Accelerator Conference, San Sebastián, Spain (EPS-AG, Spain, 2011).

[15] N. Mariani, Ph.D. dissertation, Politecnico di Milano, Milano, Italy, 2014.

[16] A. Bertarelli et al., in Presentation during the Joint WP2WP5 Meeting \& 46th Collimation Upgrade Specification Meeting, https://lhc-collimation-upgrade-spec.web.cern.ch/ lhc-collimation-upgrade-spec/Files/meetings/46/Bertarelli_ HRMT_Tests_Overview_141003.pdf.

[17] W. J. Parker, R. J. Jenkins, C. P. Butler, and G. L. Abbott, Flash method of determining thermal diffusivity, heat capacity and thermal conductivity, J. Appl. Phys. 32, 1679 (1961).

[18] H. S. Carslaw and J. C. Jaeger, Conduction of Heat in Solids, 2nd ed. (Oxford University Press, Oxford, England, 1959).

[19] American Society for Testing and Materials International Report No. ASTM E1461-13, 2013.

[20] F. P. Incropera et al., Introduction to Heat Transfer, 5th ed. (John Wiley \& Sons, Inc., New York, 2007).

[21] American Society for Testing and Materials International, ASTM Report No. E1269-11, 2011.

[22] American Society for Testing and Materials International, ASTM Report No. E228-11, 2011.

[23] A. F. Bower, Applied Mechanics of Solids (CRC Press, Boca Raton, 2009).

[24] American Society for Testing and Materials International, ASTM Report No. C1161-02c, 2002.

[25] American Society for Testing and Materials International, ASTM Report No. C695-91, 2010.

[26] American Society for Testing and Materials International, ASTM Report No. C1259-01, 2001.

[27] American Society for Testing and Materials International, ASTM Report No. C747-93, 2010. 
[28] S. Spinner et al., A comparison of experimental and theoretical relations between Young's modulus and the flexural and longitudinal resonance frequencies of uniform bars, J. Research National Bureau Standards A 64A, (1960), p. 147.

[29] S. Spinner and W. E. Tefft, A method for determining mechanical resonance frequencies and for calculating elastic moduli from these frequencies, Proceedings of the 64th Annual Meeting of the American Society of Testing and Materials 61, 1221 (1961).

[30] G. Pickett, Equations for computing elastic constants from flexural and torsional resonant frequencies of vibration of prisms and cylinders, Proc. Am. Soc. Testing Materials International 45, 846 (1945).

[31] T. Lauwagie et al., Mixed numerical-experimental identification of elastic properties of orthotropic metal plates, Independent Nondestructive Testing and Evaluation International 36, 487 (2003).

[32] American Society for Testing and Materials International, ASTM Report No. E494-10, 2010.

[33] B. Hosten, Stiffness matrix invariants to validate the characterization of composite materials with ultrasonic methods, Ultrasonics 30, 365 (1992).
[34] M. Grediac and P. A. Paris, Direct identification of elastic constants of anisotropic plates by modal analysis: Theoretical and numerical aspects, J. Sound Vibration 195, 401 (1996).

[35] C. Kohlhauser and C. Hellmich, Determination of Poisson's ratios in isotropic, transversely isotropic and orthotropic materials by means of combined ultrasonicmechanical testing of normal stiffnesses: Application to metals and wood, Eur. J. Mechanics A Solids 33, 82 (2012).

[36] D. E. Goldberg, Genetic Algorithm in Search, Optimization and Machine Learning, 1st ed. (Addison-Wesley Publishing Company Inc., 1989).

[37] M.F. Markham, Measurement of the elastic constants of fibre composites by ultrasonics, Composites 1, 145 (1970).

[38] F. Carra, A. Bertarelli, A. Dallocchio, L. Gentini, P. Gradassi, G. Maitrejean, A. Manousos, N. Mariani, N. Mounet, E. Quaranta, S. Redaelli, and V. Vlachoudis, in Proceedings of the 5th International Particle Accelerator Conference, IPAC14, Dresden, Germany (JACoW, Geneva, Switzerland, 2014). 